\title{
ITD Double-Edge Diffraction for Complex Source Beam Illumination
}

\author{
Alberto Toccafondi, Senior Member, IEEE, Stefano Mihai Canta, Member, IEEE \\ Danilo Erricolo, Senior Member, IEEE,
}

\begin{abstract}
A new high-frequency Incremental Theory of Diffraction (ITD) formulation for the double diffraction by metallic wedges when illuminated by Complex Source Points (CSP) is provided. The main motivation is the extension of the class of problems that can be studied using asymptotic (i.e. ray-based and incremental) methods by providing a double diffraction description for CSP, which are considered because they are efficient to analyze electrically large structures. The new formulation provides an accurate asymptotic description of the interaction between two edges in an arbitrary configuration, including slope diffraction contributions. Advantages of the ITD formulation for CSP illumination include avoiding the typical ray-caustic impairments of the GTD/UTD ray techniques and not requiring ray tracing in complex space. Numerical results are presented and compared to a Method-ofMoments analysis to demonstrate the accuracy of the solution.
\end{abstract}

Index Terms - Incremental Theory of Diffraction, Incremental Theory of Double Diffraction, Geometrical Theory of Diffraction, Diffraction, Complex Source Point, Complex Source Beams, Method of Moments

\section{INTRODUCTION}

$\mathbf{E}$ FFICIENT techniques to represent the illuminating field are often employed in the accurate prediction of the far field radiated or scattered by large structures, such as large reflector antennas. As an example, the ComplexSource-Beam (CSB) expansion is successfully used to represent the radiation from a given realistic source, such as a feed or a feed array, as well as a more general arbitrary directional wave field. An exact representation of scalar fields in terms of CSP launched from a point was first proposed in [1]. An alternative formulation for vector electromagnetic fields has been proposed in [2]-[4], where the field is expanded in terms of a magnetic and an electric CSP-dipole distribution on a sphere enclosing the actual sources. The expansion coefficients may be obtained by matching the CSB expansion to the known far-field pattern of the feed, or to its vector spherical-wave representation obtained from measured data. More recently, Martini and Maci [5], [6] have proposed a non-redundant

Toccafondi is with the Dipartimento di Ingegneria dell'Informazione, Università di Siena, Via Roma 56, 53100 Siena, Italy. Email: albertot@dii.unisi.it

Canta is with Space Systems/Loral, Palo Alto, CA 94043, USA. Email: cantas@ssd.loral.com

Erricolo is with the Department of Electrical and Computer Engineering, University of Illinois at Chicago, Chicago, IL 60607 USA. Email: derric1@uic.edu.
CSB representation that has the advantage of minimizing the number of beams and of directly computing the expansion coefficients from the known vector spherical-wave representation. In the application of these techniques, the actual source is replaced with a discrete set of CSBs, whose beams are radially directed from the enclosing sphere [3]. Each complex beam reaches a large object where it may undergo either a reflection or a diffraction by either the surface or the edges, respectively. The overall radiation can be estimated from the superposition of the contributions from only those CSBs that are strongly intercepted by the structure. Thus, a CSP field representation may provide a very efficient tool to estimate the fields radiated by large objects [7], when combined with analytic continuation in complex space of typical ray-techniques such as the Uniform Theory of Diffraction (UTD) [8].

On the other hand, when dealing with large structures, many existing electromagnetic codes resort to Physical Optics (PO) for any type of arbitrary illumination. However, it is well known that the PO approach does not produce accurate field predictions, particularly at sidelobe directions or at shadow zones [9]. To compensate for these inaccuracies, a correction term is added to the PO field estimate by performing a line integration of an incremental fringe field along the edges of the structure. Several techniques have been proposed to derive these elementary fringe contributions, based on PTD [10]-[12], EEW/ILDC [13]-[15] and ITD [16]-[18] for real source illumination. In the last years, an ITD formulation for CSB illumination has been proposed [19] which overcomes both the typical ray-caustic impairments of the GTD/UTD ray techniques and the difficulties of ray tracing in complex space. To augment the accuracy and the efficiency of PO, a fringe formulation of the field diffracted by edges in planar Perfect Electric Conductors (PEC) illuminated by a CSB has been recently presented [20], [21]. There, the total spurious effects due to the edges of the object are corrected by adiabatically distributing and integrating the local incremental fringe field coefficients along the lines of the edges.

However, for certain configurations where more than one metallic edge is present, and for grazing aspects of incidence and/or observation, the effects of multiple interactions between edges can not be overlooked, whether the wedges are either located on the same plane, or they are skewed with respect to each other, or they share a common PEC face [22]-[32]. In fact, at high frequency the dominant 
field associated with a single-diffracted ray is discontinuous due to shadowing by a second wedge. Then, the introduction of a double-diffracted field becomes necessary to compensate for discontinuities occurring mostly at grazing incidence and observation aspects for the diffracted field.

The novelty of this work is the introduction of an incremental double-diffraction formulation for CSP illumination, with the aim to overcome the problem of the discontinuities in the first-order incremental diffracted field contributions under CSP illumination of complex structures. ITD double diffraction coefficients are derived by solving a proper canonical problem for the ITD applied to Complex Source Points [19].

As pointed out in other existing UTD formulations for real source illumination [23], [25], [26], [29], [33], [34], the cascaded application of ordinary UTD coefficients fails when the edge of the second wedge lies within the transition region of the field diffracted by the first wedge. This behavior is due to the rapid spatial variation and to the non ray-optical behavior of the field diffracted by the first edge when it illuminates the second wedge. The same limitation is expected to affect the ITD representation [18], [19], [21], [35], thus preventing a simple cascaded application of the ITD coefficients for single diffraction. Therefore, it is necessary to develop an ITD double-diffraction coefficient that uniformly accounts for the different transitions that may occur. Hence, a formulation based on the application of the reaction principle for the scattering by metallic object is proposed. Equivalent filamentary currents are first introduced; in free space such currents radiate a field that is equivalent to the incremental single-diffracted field by one edge. Then the incremental double-diffracted field is represented as the reaction between the incremental field diffracted by one of the wedges and the filamentary current sources associated with the diffraction by the other wedge. The total double-diffracted field representation requires a two-fold numerical integration in the space domain on each edge of the complex structures. This formulation provides an accurate asymptotic description of the interaction between two edges, which is valid both for skewed separate wedges and for edges joined by a common PEC face. It also explicitly satisfies reciprocity and includes a double incremental slope diffraction augmentation, which provides the correct dominant high-frequency incremental contribution at grazing aspect of incidence and observation.

This article is organized as follows. Section II focuses on the similarities between the UTD and the ITD approach to obtain a double diffraction formulation. Section III presents results for two geometries with interacting edges: a square plate and a disc. All the derivations in this article are carried out for time-harmonic fields at the angular frequency $\omega$; the time convention $\exp (j \omega t)$ is assumed and suppressed throughout.

\section{Incremental Double Diffraction FORMULATION}

The geometry of the problem consists of a pair of wedges with curved edges $l_{1}$ and $l_{2}$, arranged in an ar-

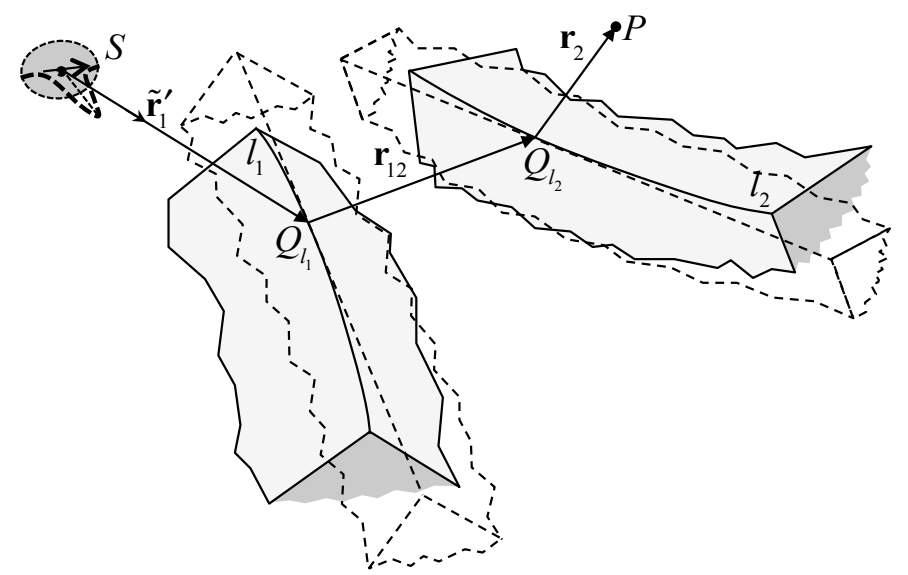

Fig. 1. Geometrical configuration for the Incremental Theory of Double Diffraction.

bitrary configuration as in Fig. 1, and illuminated by a CSP incident beam. The edges may or may not be coplanar, and they may or may not share a common face. The double diffracted field between the two wedges is represented in incremental form on the basis of the ITD formulation for single CSP diffracted fields [19]. In principle, the incremental diffracted field arising at each local point $Q_{l_{1}}$, illuminated by a CSP beam, impinges on each local point $Q_{l_{2}}$ on the second edge $l_{2}$, where it causes a second incremental diffracted field. At high frequency, it is supposed that both the wedges and the local radius of curvature of the two edges $l_{1}$ and $l_{2}$ are electrically large . Then, the incremental fields at each local point $Q_{l_{1}}$ and $Q_{l_{2}}$ may be obtained as those arising from two locally tangent canonical infinite wedges, as sketched out with dashed lines in Fig. 1.

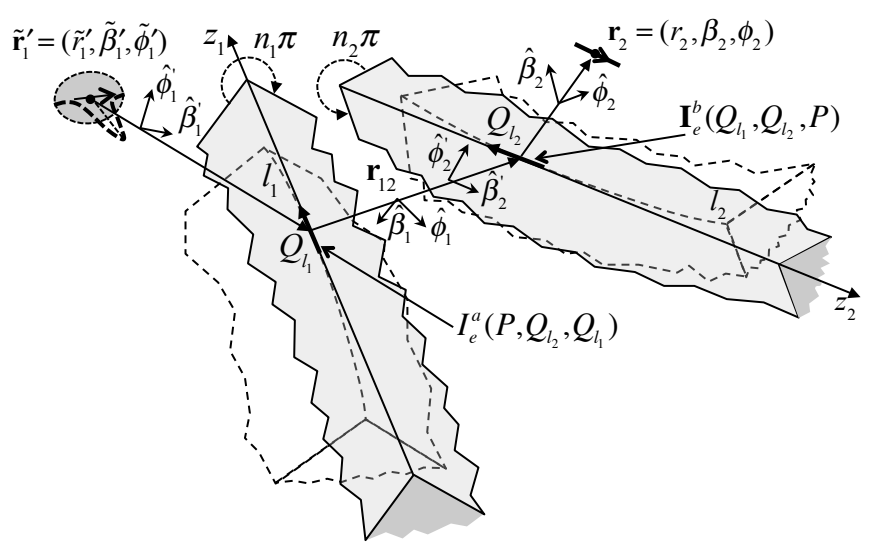

Fig. 2. The canonical configuration for the Incremental Theory of Double Diffraction.

It is now useful to refer to four local spherical edge-fixed coordinate systems: $\left(\hat{r}_{1}^{\prime}, \hat{\beta}_{1}^{\prime}, \hat{\phi}_{1}^{\prime}\right)$ and $\left(\hat{r}_{12}, \hat{\beta}_{1}, \hat{\phi}_{1}\right)$ associated with the incident and diffracted rays at $Q_{l_{1}}$ on $l_{1}$, respectively; $\left(\hat{r}_{12}, \hat{\beta}_{2}^{\prime}, \hat{\phi}_{2}^{\prime}\right)$ and $\left(\hat{r}_{2}, \hat{\beta}_{2}, \hat{\phi}_{2}\right)$ associated with the incident and diffracted rays at $Q_{l_{2}}$ on $l_{2}$. Using these local reference systems, $S \equiv\left(\tilde{r}_{1}^{\prime}, \tilde{\beta}_{1}^{\prime}, \tilde{\phi}_{1}^{\prime}\right)$ denotes the complex coordinates of the illuminating CSP with respect to $Q_{l_{1}}$ 
and $P \equiv\left(r_{2}, \beta_{2}, \phi_{2}\right)$ the coordinates of the observation point with respect to $Q_{l_{2}}$. The double diffraction mechanism between edges $l_{1}$ and $l_{2}$ can be associated with the incremental ray-path contribution $S-Q_{l_{1}}-Q_{l_{2}}-P$, where $\left(Q_{l_{1}}, Q_{l_{2}}\right)$ are all the possible pairs of incremental diffraction points along $l_{1}$ and $l_{2}$, respectively. The total double-diffracted field $\mathbf{E}_{12}^{d d}(P)$ at $P$ is then reconstructed by superimposing in integral form all the incremental double-diffracted field contributions $d \mathbf{E}_{12}^{d d}\left(P, Q_{l_{2}}, Q_{l_{1}}\right)$ at $P$, and namely

$$
\mathbf{E}_{12}^{d d}(P)=\int_{l_{1}} \int_{l_{2}} d \mathbf{E}_{12}^{d d}\left(P, Q_{l_{2}}, Q_{l_{1}}\right)
$$

It is obvious that a complete double diffraction description of the phenomena includes also the corresponding incremental ray-path contribution $S-Q_{l_{2}}-Q_{l_{1}}-P$ between edges $l_{2}$ and $l_{1}$. However, this contribution is not considered here, since it is easily obtained by swapping $Q_{l_{1}}$ with $Q_{l_{2}}$.

\section{A. Incremental formulation}

According to a well-established locality principle for high-frequency phenomena, the double-diffracted incremental field contribution $d \mathbf{E}_{12}^{d d}\left(P, Q_{l_{2}}, Q_{l_{1}}\right)$ is deduced from the canonical problem of double diffraction between two infinite uniform cylindrical wedges locally tangent at $Q_{l_{1}}$ and $Q_{l_{2}}$, with exterior angles $n_{1} \pi$ and $n_{2} \pi$, respectively, as shown in Fig. 2. To obtain a useful expression of the double-diffracted field contribution $d \mathbf{E}_{12}^{d d}\left(P, Q_{l_{2}}, Q_{l_{1}}\right)$, we refer to a particular formulation of the reaction principle for the scattering by metallic objects. Accordingly, we start by representing the incremental field at an observation point $P$ diffracted by a canonical metallic wedge, with straight edge $l$ illuminated by a source at $S$, as the field radiated in free space by a set of filamentary equivalent electric $(e)$ and magnetic $(m)$ current sources $I_{e, m}\left(P, Q_{l_{i}}, S\right)$ located at each incremental point $Q_{l_{i}}, i=1,2$, and flowing along the line $l$ of the edge. Next, we consider the canonical metallic wedge tangent at $Q_{l_{2}}$ as the scatterer, which is illuminated by two set of sources. One is the equivalent filamentary current sources distributed on the edge $l_{1}$ (labeled as $a$ ) that radiate the field diffracted by the first wedge; the other is a local $\hat{z}$ directed unit dipole located at the observation point $P$ (labeled as $b$ ). Limiting the analysis to the field generated by the equivalent filamentary electric currents $\mathbf{I}_{e}$ flowing along the canonical edges and by the illuminating electric dipoles, the reaction principle states that

$$
\begin{aligned}
& \mathbf{E}_{12}^{d d}(P)=\int_{-\infty}^{\infty}\left[\mathbf{E}_{i}^{b}\left(Q_{l_{2}}\right) \cdot \mathbf{I}_{e}^{a}\left(P, Q_{l_{2}}, Q_{l_{1}}\right)\right] d z_{2}= \\
& =\int_{-\infty}^{\infty}\left[\mathbf{E}_{i}^{a}\left(Q_{l_{2}}\right) \cdot \mathbf{I}_{e}^{b}\left(Q_{l_{1}}, Q_{l_{2}}, P\right)\right] d z_{2},
\end{aligned}
$$

where $\mathbf{E}_{i}^{a}\left(Q_{l_{2}}\right)$ is the diffracted field incident at $Q_{l_{2}}$ due to the equivalent currents at $Q_{l_{1}}, \mathbf{I}_{e}^{a}\left(P, Q_{l_{2}}, Q_{l_{1}}\right)$ is the equivalent free-space electric currents on $l_{2}$ that radiate the diffracted field at $P$ when the second wedge is illuminated by $\mathbf{E}_{i}^{a}\left(Q_{l_{2}}\right), \mathbf{E}_{i}^{b}\left(Q_{l_{2}}\right)$ is the incident field at $Q_{l_{2}}$ due to a local $\hat{z}$-directed unit electric dipole placed at $P$, and $\mathbf{I}_{e}^{b}\left(Q_{l_{1}}, Q_{l_{2}}, P\right)$ is the equivalent free-space currents on $l_{2}$ that radiate the diffracted field at $Q_{l_{1}}$ when the second wedge is illuminated by $\mathbf{E}_{i}^{b}\left(Q_{l_{2}}\right)$. The analysis is limited to the local $\hat{z}$-component of the electric field (soft component), although a similar procedure can be repeated for the local $\hat{z}$-component of the magnetic field (hard component).

To apply the reaction principle in (2), it is supposed that the single-diffracted field by the first edge $\mathbf{E}_{i}^{a}\left(Q_{l_{2}}\right)$ is generated by equivalent filamentary free-space sources distributed along the edge $l_{1}$ that radiate in the presence of the second wedge. The component of the above incremental field aligned along $\hat{\beta}_{2}^{\prime}$ (in the $Q_{l_{2}}$ reference system) is

$$
E_{i, \beta_{2}^{\prime}}^{a}\left(Q_{l_{2}}\right)=\mathbf{M}_{\beta}\left(\gamma_{12}\right) \cdot \mathbf{E}_{i}^{a}\left(Q_{l_{2}}\right),
$$

where $\mathbf{M}_{\beta}\left(\gamma_{12}\right)$ is the first row of the transformation matrix $\underline{M}\left(\gamma_{12}\right)$ between the two local spherical systems at $Q_{l_{1}}$ and $Q_{l_{2}}$, and $\gamma_{12}$ is the rotation angle defined between the ray-fixed unit vectors $\left(\hat{\beta}_{1}, \hat{\phi}_{1}\right)$ and $\left(\hat{\beta}_{2}^{\prime}, \hat{\phi}_{2}^{\prime}\right)$, as illustrated in [26]. As a consequence, the $\hat{z}_{l_{2}}$-component of the incident field at $Q_{l_{2}}$ is then

$$
\begin{aligned}
& E_{i, z_{l_{2}}}^{a}\left(Q_{l_{2}}\right)=E_{i, \beta_{2}^{\prime}}^{a}\left(Q_{l_{2}}\right)\left(\hat{\beta}_{2}^{\prime} \cdot \hat{z}_{l_{2}}\right)= \\
& =\sin \beta_{2}^{\prime} \mathbf{M}_{\beta}\left(\gamma_{12}\right) \cdot \int_{-\infty}^{\infty} \underline{\underline{D}}\left(\tilde{\nu}_{1} ; \phi_{1}, \tilde{\phi}_{1}^{\prime}\right) \cdot \mathbf{E}^{i}\left(Q_{l_{1}}\right) \frac{e^{-j k r_{12}}}{2 \pi r_{12}} d z_{1},
\end{aligned}
$$

where $\mathbf{E}^{i}\left(Q_{l_{1}}\right)$ is the incident field at $Q_{l_{1}}$ by the CSP at $S$ and

$$
\begin{aligned}
& \underline{\underline{D}}\left(\tilde{\nu}_{1} ; \phi_{1}, \tilde{\phi}_{1}^{\prime}\right)= \\
& =D_{s}\left(\tilde{\nu}_{1} ; \phi_{1}, \tilde{\phi}_{1}^{\prime}\right) \hat{\beta}_{1} \hat{\beta}_{1}^{\prime}+D_{h}\left(\tilde{\nu}_{1} ; \phi_{1}, \tilde{\phi}_{1}^{\prime}\right) \hat{\phi}_{1} \hat{\phi}_{1}^{\prime}
\end{aligned}
$$

is the incremental diffraction dyadic. Explicit expressions of $D_{s}$ and $D_{h}$ with the corresponding arguments are given in [19].The above field component is parallel to the local equivalent filamentary current source $I_{e, z_{l_{2}}}^{b}\left(Q_{l_{1}}, Q_{l_{2}}, P\right)$ at $Q_{l_{2}}$, that radiates at $Q_{l_{1}}$ the incremental field

$$
\begin{aligned}
& E_{z_{l_{2}}}^{b}\left(Q_{l_{1}}\right)=E_{\beta_{2}^{\prime}}^{b}\left(Q_{l_{1}}\right)\left(\hat{\beta}_{2}^{\prime} \cdot \hat{z}_{l_{2}}\right)= \\
& =j k \zeta \sin \beta_{2}^{\prime} \frac{e^{-j k r_{12}}}{4 \pi r_{12}} I_{e, z_{l_{2}}}^{b}\left(Q_{l_{1}}, Q_{l_{2}}, P\right) d z_{2} \sin \beta_{2}^{\prime} .
\end{aligned}
$$

Since the latter represents the incremental field at $Q_{l_{1}}$ single-diffracted by the edge $l_{2}$ when illuminated by the unit electric dipole in $P$, we obtain

$$
I_{e, z_{l_{2}}}^{b}\left(Q_{l_{1}}, Q_{l_{2}}, P\right)=\frac{2 D_{s}\left(-\nu_{2} ; \phi_{2}^{\prime}, \phi_{2}\right)\left[\mathbf{E}_{i}^{b}\left(Q_{l_{2}}\right) \cdot \hat{\beta}_{2}\right]}{j k \zeta \sin \beta_{2}^{\prime}} .
$$

By using (4) and (7), the integral in (2) can be written 
as

$$
\begin{aligned}
& \int_{-\infty}^{\infty} E_{i, z l_{2}}^{a}\left(Q_{l_{2}}\right) I_{e, z_{l_{2}}}^{b}\left(Q_{l_{1}}, Q_{l_{2}}, P\right) d z_{2}= \\
& =\int_{-\infty}^{\infty} \int_{-\infty}^{\infty} \mathbf{M}_{\beta}\left(\gamma_{12}\right) \cdot \underline{\underline{D}}\left(\tilde{\nu}_{1} ; \phi_{1}, \tilde{\phi}_{1}^{\prime}\right) \cdot \mathbf{E}^{i}\left(Q_{l_{1}}\right) \\
& \times \frac{e^{-j k r_{12}}}{2 \pi r_{12}} \sin \beta_{2} \frac{e^{-j k r_{2}}}{2 \pi r_{2}} D_{s}\left(-\nu_{2} ; \phi_{2}^{\prime}, \phi_{2}\right) d z_{1} d z_{2} .
\end{aligned}
$$

A similar procedure exists for the hard polarization. When the notation is compacted in matrix form, the final formulation is

$$
\begin{aligned}
\mathbf{E}_{12}^{d d}(P)=\int_{-\infty}^{\infty} \int_{-\infty}^{\infty} & \underline{D_{12}^{d d}}\left(\tilde{\nu}_{1}, \nu_{2} ; \tilde{\phi}_{1}^{\prime}, \phi_{1}, \phi_{2}^{\prime}, \phi_{2}\right) \cdot \mathbf{E}^{i}\left(Q_{l_{1}}\right) \\
& \times \frac{e^{-j k r_{12}}}{2 \pi r_{12}} \frac{e^{-j k r_{2}}}{2 \pi r_{2}} d z_{2} d z_{1} .
\end{aligned}
$$

Comparing the previous equation with (1), the incremental double-diffracted field contribution at $P$ is

$$
\begin{aligned}
& d \mathbf{E}_{12}^{d d}\left(P, Q_{l_{2}}, Q_{l_{1}}\right)=\underline{\underline{D}}_{12}^{d d}\left(\tilde{\nu}_{1}, \nu_{2} ; \tilde{\phi}_{1}^{\prime}, \phi_{1}, \phi_{2}^{\prime}, \phi_{2}\right) \cdot \mathbf{E}^{i}\left(Q_{l_{1}}\right) \\
& \times \frac{e^{-j k r_{12}}}{2 \pi r_{12}} \frac{e^{-j k r_{2}}}{2 \pi r_{2}} d z_{2} d z_{1}
\end{aligned}
$$

which contains the double diffraction dyadic

$$
\begin{aligned}
& \underline{\underline{D}}_{12}^{d d}\left(\tilde{\nu}_{1}, \nu_{2} ; \tilde{\phi}_{1}^{\prime}, \phi_{1}, \phi_{2}^{\prime}, \phi_{2}\right)= \\
& =\underline{\underline{D}}\left(-\nu_{2} ; \phi_{2}^{\prime}, \phi_{2}\right) \cdot \underline{\underline{M}}\left(\gamma_{12}\right) \cdot \underline{\underline{D}}\left(\tilde{\nu}_{1} ; \phi_{1}, \tilde{\phi}_{1}^{\prime}\right),
\end{aligned}
$$

and, similarly to $(5)$,

$$
\begin{aligned}
& \underline{\underline{D}}\left(-\nu_{2} ; \phi_{2}^{\prime}, \tilde{\phi}_{2}\right)= \\
& =D_{s}\left(-\nu_{2} ; \phi_{2}^{\prime}, \tilde{\phi}_{2}\right) \hat{\beta}_{2} \hat{\beta}_{2}^{\prime}+D_{h}\left(-\nu_{2} ; \phi_{2}^{\prime}, \tilde{\phi}_{2}\right) \hat{\phi}_{2} \hat{\phi}_{2}^{\prime} .
\end{aligned}
$$

The total high-frequency diffracted field by two arbitrary wedges with edges $l_{1}$ and $l_{2}$ is then obtained by distributing and integrating the incremental contribution in (10) as shown in (1).

\section{B. Even and odd spectral contributions}

As in the case of real sources illuminating the wedges [25], [26], it is best to retain in the spectral formulation the product of both the even and the odd parts of each cotangent term associated with the spectral Green's Function of a single wedge [36]. This procedure gives a local double diffraction description that provides, when integrated, the proper asymptotic order in all the different transitions that may occur. As a result, the nonvanishing contributions to the solution for the canonical problem of a pair of wedges are provided by the even parts of the final spectra, i.e. the even parts of $\underline{\underline{D}}_{12}^{d d}$. The latter are obtained from the product of both the two even and odd parts of each cotangent term in the ITD formulation [19]. On the other hand, the mixed terms that result from products between an even and an odd part of the spectrum provide a vanishing contribution when integrated into the symmetrical spectral domain. The final form of the dyadic double diffraction coefficient is

$$
\begin{aligned}
& \underline{\underline{D}}_{12}^{d d}\left(\nu_{2}, \tilde{\nu}_{1} ; \phi_{2}, \phi_{2}^{\prime}, \phi_{1}, \tilde{\phi}_{1}\right)= \\
& \underline{\underline{D_{1}}} e, e\left(\nu_{2}, \tilde{\nu}_{1} ; \phi_{2}, \phi_{2}^{\prime}, \phi_{1}, \tilde{\phi}_{1}\right)+\underline{\underline{D}}_{12}^{o, o}\left(\nu_{2}, \tilde{\nu}_{1} ; \phi_{2}, \phi_{2}^{\prime}, \phi_{1}, \tilde{\phi}_{1}\right),
\end{aligned}
$$

where

$$
\begin{aligned}
& \underline{\underline{D}}_{12}^{e, e}\left(\nu_{2}, \tilde{\nu}_{1} ; \phi_{2}, \phi_{2}^{\prime}, \phi_{1}, \tilde{\phi}_{1}\right)= \\
& \underline{\underline{D}}^{e}\left(-\nu_{2} ; \phi_{2}^{\prime}, \phi_{2}\right) \cdot \underline{\underline{M}}\left(\gamma_{12}\right) \cdot \underline{\underline{D}}^{e}\left(\tilde{\nu}_{1} ; \phi_{1}, \tilde{\phi}_{1}^{\prime}\right),
\end{aligned}
$$

is the even part of the ITD double diffraction coefficient and

$$
\begin{aligned}
& \underline{\underline{D}}_{12}^{o, o}\left(\nu_{2}, \tilde{\nu}_{1} ; \phi_{2}, \phi_{2}^{\prime}, \phi_{1}, \tilde{\phi}_{1}\right)= \\
& \underline{\underline{D}}^{o}\left(-\nu_{2} ; \phi_{2}^{\prime}, \phi_{2}\right) \cdot \underline{\underline{M}}\left(\gamma_{12}\right) \cdot \underline{\underline{D}}^{o}\left(\tilde{\nu}_{1} ; \phi_{1}, \tilde{\phi}_{1}^{\prime}\right)
\end{aligned}
$$

is the odd part. It is worth pointing out that expression (10) together with (13)-(15) is not a merely analytic continuation in complex space of that for real illuminating sources [26]. Here, the presence of the two terms $\tilde{\nu}_{1}$ and $-\nu_{2}$ assures the symmetrical behavior of the incremental double diffraction contribution with respect to the source and observation coordinates, and when (10) is integrated along the actual structure, provides the correct dominant high-frequency contribution at grazing aspect of incidence and observation.

Because of space limitations, the attention was focused on the derivation of the incremental double contributions. Considerations analogous to those in [26] are valid for singularity and discontinuity contributions in the representation of the total double-diffracted field and will be the subject of future investigation.

\section{Numerical Results}

\section{A. Perfectly conducting square plate}

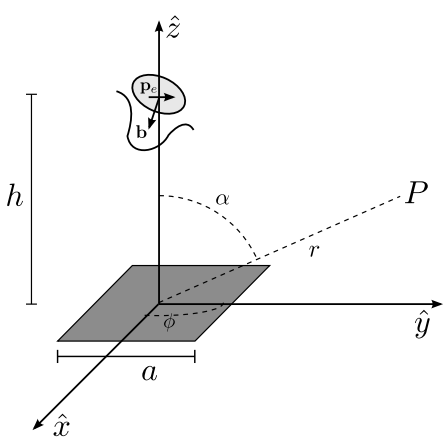

Fig. 3. Geometry for the scattering by a square plate illuminated by a CSP.

Consider the scattering from a perfectly conducting square plate illuminated by a single CSP, as shown in Fig. 3. A $\hat{y}$-directed electric CSP dipole is placed at $(0,0, h=8 \lambda)$ above a square plate with sides $a=5 \lambda$, and located in the $x y$ plane. The vector associated with the beam is $\mathbf{b}=\lambda(\sin (8 \pi / 9), 0, \cos (8 \pi / 9))$, and the beam points towards one of the edges of the plate. Both 
$\hat{\theta}$ - and $\hat{\phi}$-components of the scattered electric field have been calculated for observations at a distance $r=11 \lambda$ and presented in Fig. 4 for the E-plane and Fig. 5 for the H-plane, respectively. Numerical results obtained from the Physical Optics (PO) method, the incremental fringe method $(\mathrm{PO}+\mathrm{IFF})$ computed as in $[21]$ and the present method $(\mathrm{PO}+\mathrm{IFF}+\mathrm{DD})$ are plotted for $\alpha \in[0,2 \pi]$ and compared with a method of moment (MoM) solution.

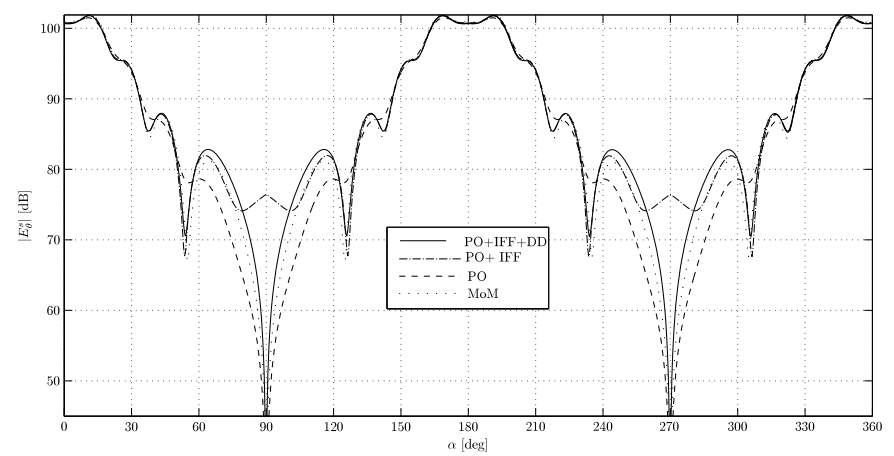

Fig. 4. $\left|E_{\theta}^{S}\right|$ component of the scattered electric field for the square plate illuminated by a single CSP electric dipole. Observations are made in the E-plane.

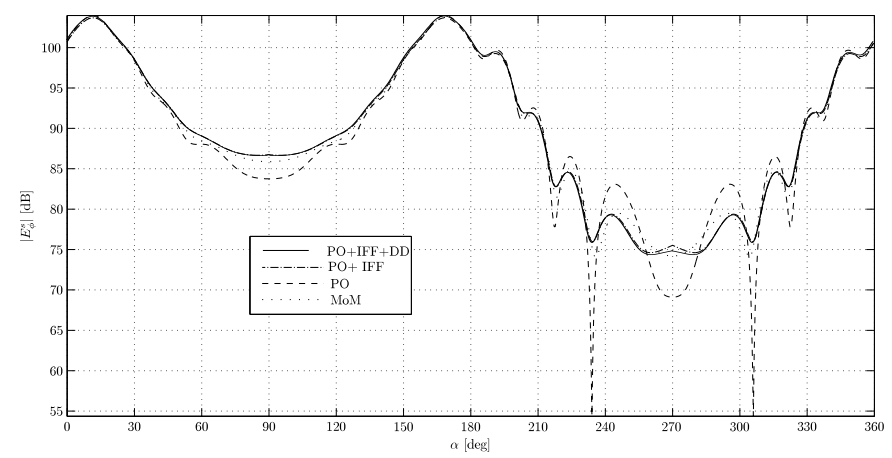

Fig. 5. $\left|E_{\phi}^{s}\right|$ component of the scattered electric field for the square plate illuminated by a single CSP electric dipole. Observations are made in the H-plane.

The $\mathrm{PO}+\mathrm{IFF}$ curve is in good agreement with MoM calculations, except for the region close to grazing observation. As expected, at these observation aspects, the first-order ITD incremental diffraction coefficients exhibit a discontinuity that is removed with the introduction of the new ITD double diffraction contributions, as shown in Fig. 4. Additionally, the accuracy of PO calculations is significantly improved when the incremental fringe contributions are added. The same considerations also apply for the $\hat{\phi}$-component of the scattered electric field shown in Fig. 5. However, in this case, the ITD double diffraction contribution is not as important, because, at grazing observation aspects, the fringe calculation do not exhibit first-order discontinuities in the ITD field. Furthermore, correct contributions from vertices in this illumination condition become important, but they are not properly taken into consideration in this formulation since they are reconstructed only by the truncation of the IFF integration along the edges of the plate. For both polarizations, once the double diffracted field contribution is added to the $\mathrm{PO}+\mathrm{IFF}$ formulation, a much closer result to the MoM computations is achieved, especially in the region near to the $x y$-plane.

The effects of the double-diffracted field are more evident in the E-plane cut: double diffraction contributes to recover the solution for the electric field close to the $x y$-plane. In the H-plane cut, however, double diffraction plays a negligible role: we believe that the discrepancies found with the MoM are due to the fact that the corner diffraction contributions play a significant role.

\section{B. Perfectly conducting disc}

To investigate the effectiveness of the formulation when the scatterer does not include vertices, the scattering from a perfectly conducting disc illuminated by a CSP is now considered, as shown in Fig. 6. A horizontal $\hat{y}$-directed CSP dipole is placed at $(0,0, h=8 \lambda)$ above a PEC disc with radius $a=2.5 \lambda$, centered at the origin of the coordinate system and perpendicular to the $\hat{z}$ axis. The vector associated with the beam is $\mathbf{b}=\lambda(\sin (8 / 9 \pi), 0, \cos (8 / 9 \pi))$, so the beam points toward the edge of the disc. The observation is made at a distance $r=10 \lambda$ in the $y z$ plane for $0 \leq \theta \leq \pi$.

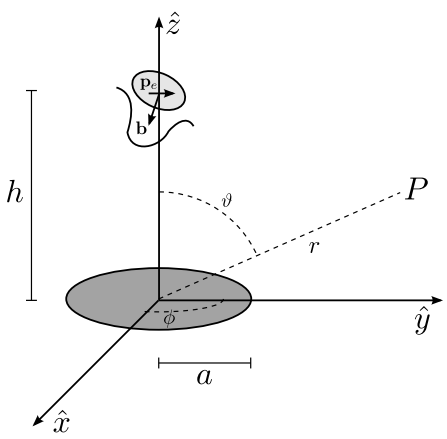

Fig. 6. Geometry for the scattering by a planar disc illuminated by a CSP.

In Fig. 7 , the $\hat{\theta}$-component of the total electric field obtained from the proposed $\mathrm{PO}+\mathrm{IFF}+\mathrm{DD}$ formulation is compared with the results from a first order (single diffracted) $\mathrm{PO}+\mathrm{IFF}$ calculation, and with a MoM prediction. Good agreement between MoM and first order $\mathrm{PO}+\mathrm{IFF}$ is found everywhere except for the region close to grazing observation, where double diffraction coefficients need to be introduced to compensate for the discontinuity of the ITD incremental diffraction field, as expected. Fig. 8 shows the good matching between $\mathrm{PO}+\mathrm{IFF}+\mathrm{DD}$ and MoM, with the compensating effect over the ITD discontinuity. To provide a better understanding of the double diffraction phenomenon, the scattered electric field $\left|E_{\theta}^{s}\right|$ is plotted in Fig. 9. It can be seen that the double diffraction component compensates the effect of the IFF field and provides a good matching with the MoM solution. 




Fig. 7. $\left|E_{\theta}\right|$ component of the total electric field for the case of a disc.

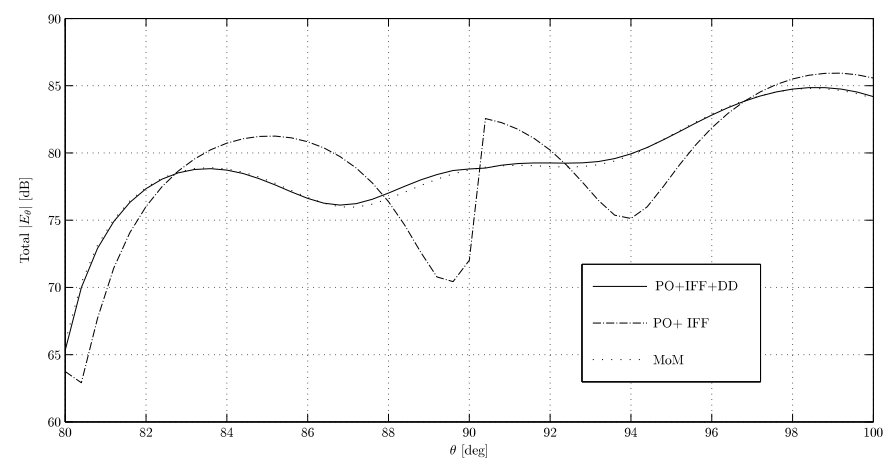

Fig. 8. Detail of the $\left|E_{\theta}\right|$ component of the total electric field for the case of a disc.

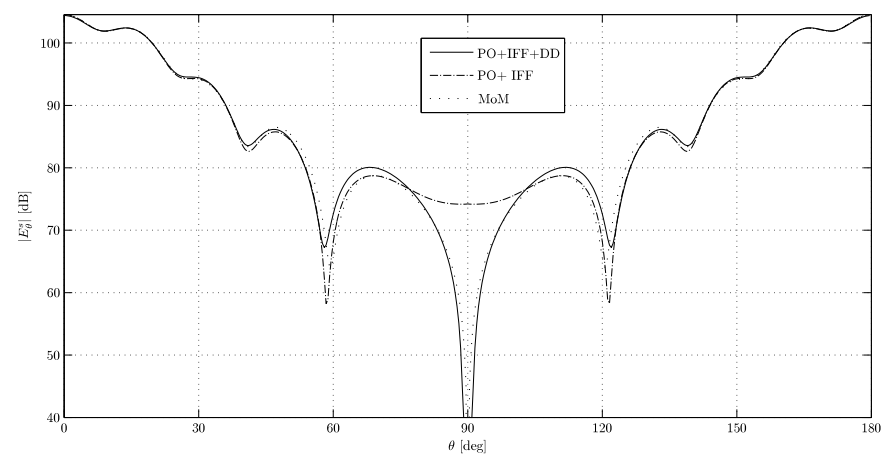

Fig. 9. $\left|E_{\theta}^{s}\right|$ component of the scattered electric field for the case of a disc.

\section{Conclusions}

A double-diffraction formulation for the incremental diffraction by two interacting wedges under CSP illumination has been introduced. The formulation has been obtained by using a reciprocal reaction analysis. In particular, the incremental double-diffracted field is represented as the reaction between the incremental field diffracted by one of the two wedges and equivalent filamentary current sources associated with the diffraction by the other wedge. The derivation of total double-diffracted field requires a two-fold numerical integration in the space domain on each edge of the complex structures. The application to the high-frequency scattering from double-edges structures has been investigated. It has been shown that the for- mulation satisfies reciprocity and, when combined with a single-diffracted field representation, provides a valid high-frequency description of the scattering by an obstacle illuminated by a CSP including grazing aspects of incidence and observation. Numerical results obtained for the scattering by a square plate and a circular disc are found in very good agreement with those calculated from a MoM solution. These simple examples have been chosen to illustrate the accuracy of the proposed method that uniformly accounts for the different transitions that may occur in reconstructing the total scattered or diffracted field.

\section{ACKnowledgements}

This work was partially supported by AFOSR Grant FA9550-05-1-0443 and AFOSR Grant FA9550-12-1-0174.

\section{REFERENCES}

[1] A. N. Norris, "Complex point-source representation of real sources and the Gaussian beam summation method," Journal of the Optical Society of America A, vol. 3, pp. 2005-2010, 1986.

[2] K. Tap, P. Pathak, and R. Burkholder, "An exact CSP beam representation for EM wave radiation," in Electromagnetics in Advanced Applications. International Conference on, September 2007, pp. $75-78$.

[3] P. Pathak, Y. Kim, R. Burkholder, and T.-H. Lee, "An efficient hybrid UTD-CSB-VWF approach for the analysis of large reflector antennas," in Electromagnetic Theory (EMTS), 2010 URSI International Symposium on, 2010, pp. 847-849.

[4] K. Tap, P. Pathak, and R. Burkholder, "Exact complex source point beam expansions for electromagnetic fields," IEEE Transactions on Antennas and Propagation, vol. 59, no. 9, pp. 3379 -3390 , sept. 2011.

[5] E. Martini, G. Carli, and S. Maci, "A non redundant complex source expansion derived by using spherical modes," in Antennas and Propagation Society International Symposium., 2009, pp. 1-4.

[6] _ "An exact non redundant Complex Source Point beam representation of an EM field obtained from a spherical wave expansion," in Electromagnetic Theory (EMTS), 2010 URSI International Symposium on, 2010, pp. 74-76.

[7] H.-T. Chou and P. H. Pathak, "Fast Gaussian beam based synthesis of shaped reflector antennas for contoured beam applications," IEE Proceedings - Microwaves, Antennas and Propagation, vol. 151, no. 1, p. 13, 2004.

[8] R. G. Kouyoumjian and P. H. Pathak, "A uniform geometrical theory of diffraction for an edge in a perfectly conducting surface," Proceedings of the IEEE, vol. 62, no. 11, pp. 14481461, 1974.

[9] P. Pathak, Antenna handbook - Theory, Application and Design. Van Nostrand Reinhold Company, 1988, ch. Techniques for highfrequency problems.

[10] P. Ya. Ufimtsev, Method of Edge Waves in the Physical Theory of Diffraction. Translation by the U.S. Air Force Foreign Technology Division, Wright-Patterson AFB, OH, released Sept., 1971, vol. 7 .

[11] — , "Elementary edge waves and the physical theory of diffraction," Electromagnetics, vol. 11, no. 2, pp. 125-160, 1991.

[12] — Fundamentals of the Physical Theory of Diffraction. Hoboken, NJ: Wiley \& Sons, Inc., 2007.

[13] A. Michaeli, "Elimination of infinities in equivalent edge currents; I: fringe components," IEEE Transactions on Antennas and Propagation, vol. 32, pp. 252-258, 1984.

[14] — , "Equivalent edge currents for arbitrary aspects of observation," IEEE Transactions on Antennas and Propagation, vol. 32, no. 3, pp. 252-258, 1984.

[15] — - "Elimination of infinities in equivalent edge currents, Part II: Physical optics components," IEEE Transactions on Antennas and Propagation, vol. 34, no. 8, 1986. 
[16] R. Tiberio and S. Maci, "An incremental theory of diffraction: scalar formulation," IEEE Transactions on Antennas and Propagation, vol. 42, no. 5, pp. 600-612, 1994.

[17] R. Tiberio, S. Maci, and A. Toccafondi, "An incremental theory of diffraction: electromagnetic formulation," IEEE Transactions on Antennas and Propagation, vol. 43, no. 1, pp. 87-96, 1995.

[18] R. Tiberio, A. Toccafondi, A. Polemi, and S. Maci, "Incremental Theory of Diffraction: A New-Improved Formulation," IEEE Transactions on Antennas and Propagation, vol. 52, no. 9, pp. 2234-2243, 2004.

[19] A. Polemi, A. Toccafondi, G. Carluccio, M. Albani, and S. Maci, "Incremental theory of diffraction for complex point source illumination," Radio Science, vol. 42, no. 6, pp. 1-13, 2007.

[20] S. M. Canta, D. Erricolo, and A. Toccafondi, "Incremental fringe formulation for the scattering of Complex Point Source beam expansion by planar metallic objects," in Antennas and Propagation Society International Symposium, 2009, pp. 1-4.

[21] _ "Incremental Fringe Formulation for a Complex Source Point Beam Expansion," IEEE Transactions on Antennas and Propagation, vol. 59, no. 5, pp. 1553-1561, May 2011.

[22] R. Tiberio and R. G. Kouyoumjian, "A uniform GTD solution for the diffraction by strips illuminated at grazing incidence," Radio Science, vol. 14, no. 6, pp. 933-941, 1979.

[23] R. Tiberio, G. Manara, G. Pelosi, and R. G. Kouyoumjian, "High-frequency electromagnetic scattering of plane waves from double wedges," IEEE Transactions on Antennas and Propagation, vol. 37, no. 9, pp. 1172-1180, 1989.

[24] F. Capolino, M. Albani, S. Maci, and R. Tiberio, "Double diffraction at a pair of coplanar skew edges," IEEE Transactions on Antennas and Propagation, vol. 45, no. 8, pp. 1219-1226, 1997.

[25] M. Albani, "A uniform double diffraction coefficient for a pair of wedges in arbitrary configuration," IEEE Transactions on Antennas and Propagation, vol. 53, no. 2, pp. 702-710, 2005.

[26] A. Toccafondi and R. Tiberio, "An incremental theory of double edge diffraction," Radio Science, vol. 42, no. 6, p. RS6S30, 2007.

[27] L. P. Ivrissimtzis and R. J. Marhefka, "Double diffraction at a coplanar skewed edge configuration," Radio Science, vol. 26, no. 4 , p. $821,1991$.

[28] _ - "A uniform ray approximation of the scattering by polyhedral structures including higher order terms," IEEE Transactions on Antennas and Propagation, vol. 40, no. 11, pp. 13021312,1992

[29] D. Erricolo, "Experimental validation of second order diffraction coefficients for computation of path-loss past buildings," vol. 44, no. 1, pp. 272-273, Feb. 2002.

[30] D. Erricolo, G. D'Elia, and P. Uslenghi, "Measurements on scaled models of urban environments and comparisons with raytracing propagation simulation," vol. 50, no. 5, pp. 727-735, May 2002.

[31] D. Erricolo, U. G. Crovella, and P. Uslenghi, "Time-domain analysis of measurements on scaled urban models with comparisons to ray-tracing propagation simulation," vol. 50, no. 5, pp. 736-741, May 2002.

[32] D. Erricolo and P. Uslenghi, "Two-dimensional simulator for propagation in urban environments," IEEE Transactions on Vehicular Technology, vol. 50, no. 4, pp. 1158-1168, July 2001.

[33] S. W. Lee, Y. Rahmat-Samii, and R. C. Menendez, "GTD, ray field and comments on two papers," IEEE Transactions on Antennas and Propagation, vol. 26, no. 2, pp. 352-354, 1978.

[34] M. Albani, F. Capolino, S. Maci, and R. Tiberio, "Diffraction at a thick screen including corrugations on the top face," IEEE Transactions on Antennas and Propagation, vol. 45, no. 2, pp. 277-283, 1997.

[35] D. Erricolo, S. M. Canta, H. T. Hayvaci, and M. Albani, "Experimental and Theoretical Validation for the Incremental Theory of Diffraction," vol. 56, no. 8, pp. 2563-2571, Aug. 2008.

[36] L. B. Felsen and N. Marcuvitz, Radiation and scattering of waves. Wiley-IEEE Press, 1994. 